\title{
Efficiency of Exciton and Charge Carrier Photogeneration in a Semiconducting Polymer
}

\author{
E. Hendry, ${ }^{1, *}$ J. M. Schins, ${ }^{2}$ L. P. Candeias, ${ }^{2}$ L. D. A. Siebbeles, ${ }^{2}$ and M. Bonn ${ }^{1,3}$ \\ ${ }^{1}$ Leiden Institute of Chemistry, Leiden University, P.O. Box 9502, 2300 RA Leiden, The Netherlands \\ ${ }^{2}$ Interfaculty Reactor Institute, Delft University of Technology, Mekelweg 15, $2629 \mathrm{JB}$ Delft, The Netherlands \\ ${ }^{3}$ FOM Institute for Atomic and Molecular Physics, Kruislaan 407, 1098 SJ, Amsterdam, The Netherlands
}

(Received 8 August 2003; published 13 May 2004)

\begin{abstract}
We determine the efficiencies for the formation of excitons and charge carriers following ultrafast photoexcitation of a semiconducting polymer (MEH-PPV). The simultaneous, quantitative determination of exciton and charge photoyields is achieved through subpicosecond studies of both the real and the imaginary components of the complex conductivity over a wide frequency range. Predominantly excitons, with near-unity quantum efficiency, are generated on excitation, while only a very small fraction $\left(<10^{-2}\right)$ of free charges are initially excited, consistent with rapid $(\sim 100 \mathrm{fs})$ hot exciton dissociation. These initial charges are very short lived, decaying on subpicosecond time scales.
\end{abstract}

DOI: 10.1103/PhysRevLett.92.196601

Since their discovery in the 1970 s, semiconducting conjugated polymers have received considerable interest owing to their potential in technological applications, particularly in electronics [1]. These materials have many advantages over conventional semiconductors: They are low cost, easy to process, lightweight and malleable, and have shown significant promise in lightemitting diodes [2], photovoltaics, and laser optics [3]. Despite their widespread optical applications, the nature of the photoexcitation physics in these materials is currently subject to intense debate [4-6]. One of the key questions that has remained controversial is whether, initially upon photoexcitation, excitons or charge carriers are primarily formed. From photoconductivity measurements [7], it is evident that free charges are formed, but the mechanism and efficiency of free charge formation remains polemical: Some studies suggest that excitons (Coulombically bound electron-hole pairs) are the primary photoexcitation product, which may dissociate into free charges after migration to electron (or hole) accepting defects, by absorption of a second photon or by bimolecular exciton-exciton annihilation $[8,9]$. Other studies suggest electron-hole pairs as the direct and predominant initial excitation product [10]. This apparent contradiction may be related to the selective sensitivity of different experimental approaches: For example, photoconductivity (PC) measurements [7] are sensitive only to free charges (responsible for real conductivity), whereas transient absorption (TA) and stimulated emission (SE) measurements [11-13] can probe excitons (bound electron-hole pairs responsible for imaginary conductivity). However, the simultaneous detection of the real and imaginary conductivity with sufficient time resolution is necessary to allow a quantitative comparison of both free and bound charges upon photoexcitation. The relatively recent technique of terahertz time domain spectroscopy [14] (THz-TDS) provides this opportunity.

In this Letter we present the first investigation of a semiconducting polymer using THz-TDS. This technique
PACS numbers: 72.80.Le, 71.35.Aa, 73.50.Gr, 73.61.Ph

allows us to monitor the evolution of free and bound charges on subpicosecond time scales following photoexcitation, through the time-dependent real and imaginary components of frequency dependent $(0.2-1.5 \mathrm{THz})$ conductivity. This allows for an unambiguous assignment of the excited species during the excitation process, helping to bridge the gap between PC and TA/SE experiments. Our results demonstrate that a very small fraction $\left(<10^{-2}\right)$ of excitations directly result in free carriers, while excitons are the dominant initial excitation product, with a quantum efficiency of the order unity.

The poly\{2-methoxy-5-(2'-ethyl-hexyloxy)- $p$ phenylene-vinylene (MEH-PPV) samples were prepared under nitrogen atmosphere using MEH-PPV from Aldrich. Solutions of the polymer in chloroform were drop cast onto quartz plates to make $\sim 20 \mu \mathrm{m}$ thick films. These are mounted in a closed cycle helium cryostat (30$300 \mathrm{~K})$ under vacuum $\left(10^{-4} \mathrm{mbar}\right)$ to minimize photooxidation [15].

The $\mathrm{THz}$ setup is similar to that in Ref. [14]. THz pulses are essentially single cycle electromagnetic pulses of about 1 ps duration. The transmitted field strength $E(t)$ is measured directly in the time domain, through electrooptical sampling with $800 \mathrm{~nm}, 150$ fs pulses: This "gating" technique provides a time resolution significantly better than the $1 \mathrm{ps} \mathrm{THz}$ pulse width [16]. Exciting MEH-PPV above the absorption gap ( $2.5 \mathrm{eV}$ [5]) using $266 \mathrm{~nm}(4.5 \mathrm{eV})$ or $400 \mathrm{~nm}(3 \mathrm{eV}), 150$ fs pulses, allows investigation of photoexcited species. We record the pump-induced modulation in the field transmission $\Delta E(t, \tau)$ as a function of delay $\tau$ between pump and $\mathrm{THz}$ probe - see inset of Fig. 1(a). $\tau=0$ is defined as the temporal overlap of the fs excitation pulse with the peak field strength of the $\mathrm{THz}$ probe pulse. At all delays the modulation depth is less than $1 \%$ of the weak, nonperturbative probe field $(\sim 1 \mathrm{kV} / \mathrm{cm})$.

Figure 1(a) shows the magnitude of $\Delta E(t, \tau)$ as a function of delay, $\tau$, for $400 \mathrm{~nm}$ excitation (fluence: $1.2 \pm$ $0.5 \times 10^{20}$ photons $/ \mathrm{m}^{2}$ ) at $30 \mathrm{~K}$. Clearly, there are two 

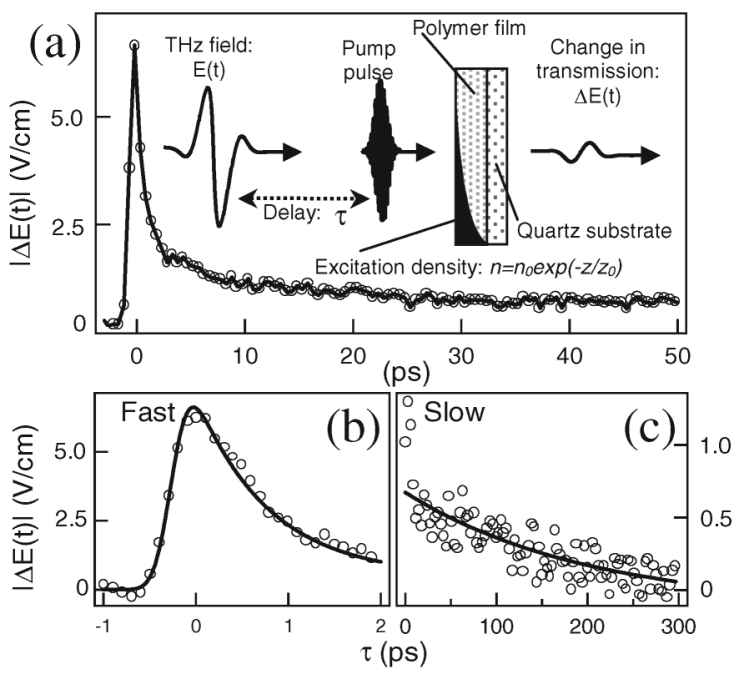

FIG. 1. (a) Inset: Photoexcitation occurs with 150 fs pulses (266 or $400 \mathrm{~nm}$ ), while the conductivity at delay $\tau$ is probed using pulsed $\mathrm{THz}$ radiation $(1 \mathrm{ps}, 0-1.5 \mathrm{THz})$. By varying the delay between pump and probe pulses and measuring the magnitude of the $\mathrm{THz}$ field modulation, $\Delta E(t, \tau)$, the photoconductivity dynamics can be studied. The dynamics of MEHPPV show two distinct decays, designated fast and slow. (b) The fast time dynamics decays exponentially with time constant $0.9 \pm 0.2 \mathrm{ps}$, while the rise time is $\sim 0.3 \mathrm{ps}$. (c) The slow time dynamics occur with a time constant of $200 \pm 40$ ps.

decay components: The fast signal decay is described well by an exponential with fast time constant $\tau_{f}=$ $0.9 \pm 0.2$ ps [Fig. 1(b)]. The rise time of the signal of $0.3 \mathrm{ps}$ is determined by the resolution of our system, consistent with previous reports [16]. The slowly decaying signal is described well by an exponential with time constant $\tau_{s}=200 \pm 40$ ps [see Fig. 1(c)]. Similar bimodal decay dynamics have been observed in PC, TA, and SE measurements on MEH-PPV and similar derivatives [11-13], while others have measured only the fast [10] or slow $[7,17]$ time dynamics because of sensitivity or resolution issues. We have performed TA measurements on our samples, probing with $800 \mathrm{~nm}$ pulses, and observed identical fast decay kinetics. The response at $800 \mathrm{~nm}$ has, however, been attributed to both excitons [13] and charges [18], so unambiguous determination of species with this experiment alone is not possible. The strength of THzTDS lies in the direct determination of the real and imaginary contributions to the conductivity at different times $\tau$ after excitation. The complex, frequency dependent conductivity $\sigma(\omega, \tau)$ can be derived from the transmitted $\mathrm{THz}$ field, $E(t)$, and the time-dependent pump-induced modulation in transmission, $\Delta E(t, \tau)$, following the experimental procedure and analysis presented in Ref. [19].

Figure 2 shows $\sigma(\omega)$ at $\tau=0.5 \mathrm{ps}(\mathrm{a})$, and $\tau=10 \mathrm{ps}$ (b), after excitation. Initially the photoconductivity has significant real and imaginary parts, both increasing with frequency-see Fig. 2(a). The real component decays on the fast time scale observed in Fig. 1, and $10 \mathrm{ps}$ after

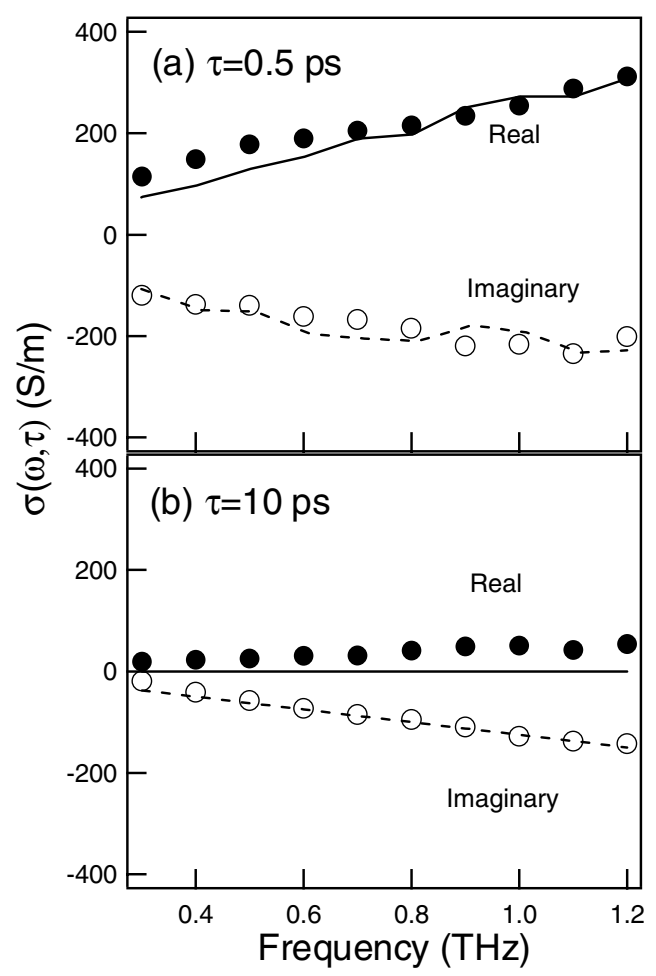

FIG. 2. (a) The complex conductivity of an MEH-PPV film measured $0.5 \mathrm{ps}$ after excitation (circles). The lines represent the conductivity of a hole charge on a PPV chain simulated using the formalism introduced in Ref. [21], for a density $n_{0}^{\text {free }} \sim 3.2 \times 10^{21} \mathrm{~m}^{-3}$ (lines). (b) The complex conductivity measured 10 ps after initial excitation (circles) and the conductivity expected for bound excitons (lines) generated with unit quantum efficiency and polarizability, $\alpha \sim 800 \AA^{3}$.

excitation the conductivity is almost completely imaginary - see Fig. 2(b). Pump-induced thermal effects are orders of magnitude smaller [20].

The complex conductivity observed at $\tau=0.5 \mathrm{ps}$ is characteristic of dispersive free charge transport in a disordered medium, where localization caused by the disorder in the material structure causes non-Drude behavior. Using the formalism introduced in Ref. [21] we can simulate the conductivity of a charge along a polymer chain. This model is based on the tight-binding approximation combined with static torsional disorder - deviations from planar alignment of the chain determining the effective conjugation length of the polymer chain. The scaled result for complex hole conductivity is plotted as lines in Fig. 2(a). Our measurement is the sum of electron and hole conductivities. We expect these to be similar, as they are limited by the same conformational restraints. Indeed, electron and hole conductivities measured at $34 \mathrm{GHz}$ are comparable [22]. Clearly, the simulation reproduces the key characteristics of our measured conductivity: Substantial real and imaginary parts increasing with frequency. This implies that conductivity on the fast time scale is dominated by free charges. These are generated promptly on the time scale 
of our experiment, i.e., within the first 300 fs. From a comparison between the data and the simulation, the free charge density at the interface $(z=0)$ is found to be $n_{0}^{\text {free }} \sim 3.2 \times 10^{21} \mathrm{~m}^{-3}$. With an excitation fluence of $\sim 1.2 \times 10^{20} \mathrm{~m}^{-2}$, a penetration depth of $\sim 100 \mathrm{~nm}$, and considering reflective pump losses, the total absorbed pump density is $n_{0}^{\text {total }} \sim 8 \times 10^{26} \mathrm{~m}^{-3}$. This suggests that only a very small fraction $\left(\sim 10^{-5}\right)$ of initial excitations results in the formation of free charges. The model is known, however, to overestimate the conductivity [21], as it neglects nontorsional defects such as conjugation breaks. The experimentally observed mobility of charge carriers at $34 \mathrm{GHz}$ in an MEH-PPV film is less than 3 orders of magnitude smaller [23] than that predicted by the model. Further, this factor is smaller at $\mathrm{THz}$ frequencies, as the $\mathrm{THz}$ mobility is less sensitive to defects. The calculated conductivity can therefore be at most 3 orders of magnitude higher than the actual value in a thin film. Thus, a conservative upper limit of the initial quantum yield of charge carriers can be set at $\sim 10^{-2}$. We cannot, from our data, identify the conductivity decay mechanism: it may be due to, e.g., charge recombination or trapping at defects in the polymer chain.

Charges generated by excitation at $400 \mathrm{~nm}(3 \mathrm{eV})$ have an initial excess energy of approximately $0.5 \mathrm{eV}$ above the absorption gap of MEH-PPV [5]. These "hot" carriers may respond to the $\mathrm{THz}$ field differently from thermalized carriers, to which the discussion above applies. However, Fig. 3 depicts the conductivity of an MEH-PPV sample containing 50\% PCBM [1-(3-methoxycarbonyl)-propyl-1-phenyl- $(6,6) \mathrm{C}_{61}$, an exciton-dissociating electron scavenger] 10 ps after photoexcitation (after cooling) revealing the conductivity of thermalized holes. The lines represent the model introduced above with a density $n_{0}^{\text {free }} \sim 8.0 \times 10^{21} \mathrm{~m}^{-3}$. The similarity of the frequency dependence in Figs. 2(a) and 3 strongly suggests we are looking at cool charge carriers in

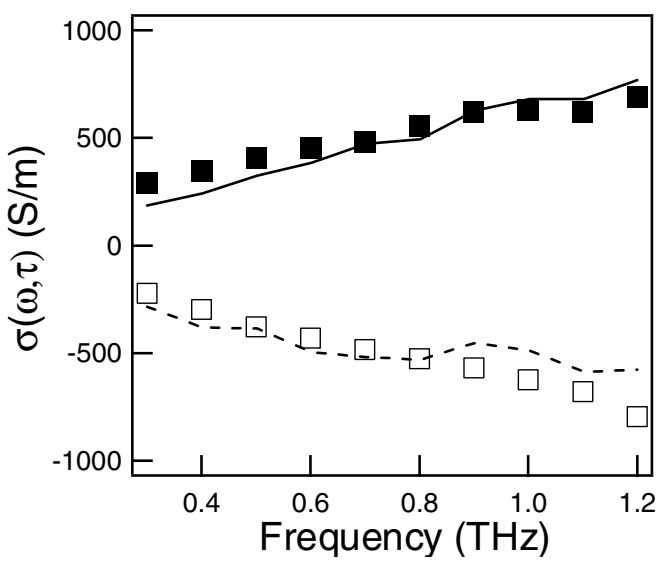

FIG. 3. The complex conductivity measured 10 ps after photoexcitation on an MEH-PPV/PCBM blended sample, demonstrating the response for thermalized hole charges. The lines represent the simulated conductivity also shown in Fig. 2(a), with a density $n_{0}^{\text {free }} \sim 8.0 \times 10^{21} \mathrm{~m}^{-3}$. both cases, since we would expect a very different conductivity for hot and cool charges - a nonthermal charge would be able to overcome many of the energy barriers that determine the simulated conductivity from Ref. [21].

After the initial fast decay (at $\tau=10 \mathrm{ps}$ ), the extracted conductivity is mostly imaginary throughout the probed frequency range - see Fig. 2(b). This is the spectral signature associated with the dielectric polarization of excitons, corresponding to temporary distortion of the exciton wave function by the field. As we are probing at frequencies $(1 \mathrm{THz}=4.1 \mathrm{meV})$ well below any exciton resonance or the exciton binding energy, the complex conductivity is determined by the dc polarizability $(\alpha)$ of the exciton. Indeed, $\sigma(\omega)$ in Fig. 2(b) can be reproduced using the Clausius-Mossotti equation [24], which relates $\alpha$ to the dielectric function $(\varepsilon)$, and to the conductivity through $\sigma(\omega)=-i \omega n \varepsilon_{0} \varepsilon$, with $n$ the exciton density. The product of the exciton density and polarizability therefore determines the fit shown in Fig. 2(b). Reports of the exciton polarizability in solid PPV films vary from $800-3000 \AA^{3}$ (see Ref. [25] for a summary). These numbers result in a value for $n$ corresponding to a quantum efficiency between 0.3 and 1 . As very few charge carriers are generated on photoexcitation, a quantum efficiency close to 1 is probable, yielding $\alpha \sim$ $800 \AA^{3}$. This is comparable to the theoretical estimate of $\sim 1000 \AA^{3}$ for an exciton on an isolated PPV chain [26], suggesting that interchain interactions do not significantly change the delocalization of the singlet exciton. An exciton polarizability of this magnitude implies a fairly weakly bound exciton (binding energy $\sim 0.8 \mathrm{eV}$ [26]), with a wave function spread over several monomer units (electron-hole mean square displacement $\sim 11 \AA$ [26], in agreement with observations on other $\pi$-conjugated systems [27]). Our results are inconsistent with more strongly bound excitons (with smaller polarizabilities that would imply a quantum efficiency exceeding 1) and more weakly bound Wannier excitons [28] [with larger polarizabilities that would imply an exciton quantum efficiency of less than $10 \%$, which is conflicting with the singlet exciton luminescence efficiency $(25 \%$ [29]) in MEH-PPV films]. Summarizing, it is clear that bound charges are the initial excitation products in MEH-PPV, with near-unity quantum efficiency, while only a small number $\left(<10^{-2}\right)$ of free charges are generated within the first $300 \mathrm{fs}$, which relax on a subpicosecond time scale.

As to the origin of the minority charge carriers, we note that for the high fluences used in our experiments, 2nd order effects may be anticipated, as the average distance between excitons is $\sim 1-2 \mathrm{~nm}$, of the order of the electron-hole mean square displacement $\sim 11 \AA$ [26]. Indeed, exciton-exciton annihilation [30], generating free charges, may explain the small real component of the slowly decaying conductivity in Fig. 2(b). However, it cannot be the origin of the charge generation at early times, since we observe a linear dependence of the signal 
on excitation fluence, consistent with Moses et al. [10] and incompatible with an exciton-exciton annihilation mechanism. Further, this mechanism requires diffusional motion, which occurs much slower than the time scale considered here.

Recent exciton models [31,32] account for fast photogeneration of charges by the rapid dissociation of singular excitons. The unrelaxed, nascent excitons (before cooling) may use the excess excitation energy (above the $S_{1}$ ground state, around $0.5 \mathrm{eV}$ for $400 \mathrm{~nm}$ ) to dissociate into free charges. This results in a small number of free and thermalized charges with a predicted $<10^{-2}$ quantum efficiency. Silva et al. [8] have included excitation to even higher energy states by a second photon within the excitation pulse, resulting in dissociation efficiencies $\sim 10^{-2}$, in agreement with our data. At high fluences, this process is linear in excitation intensity due to saturation of the initial absorption. Since exciton cooling is rapid ( $\sim 100 \mathrm{fs}$ ) [31,32], a hot exciton dissociation process explains the fast generation of free charges, within the first $300 \mathrm{fs}$, observed here.

This hot exciton dissociation mechanism is corroborated by the observed changes in the signal resulting from variation in the excitation wavelength and sample temperature. Experimentally, when corrected for the lower density of incident photons, for excitation with 266 rather than $400 \mathrm{~nm}$, the fast signal is twice as large corresponding to a larger quantum yield for the generation of free charges. Further, because the exciton dissociation energy originates from the excitation pulse rather than from thermal contributions, the hot exciton models also predict temperature independent dissociation. In our experiments, heating the sample from 30 to $300 \mathrm{~K}$ results in a small $(<20 \%)$ decrease in the signal attributed to free charges. The most likely explanation for this is a decrease in mobility, rather than density, possibly due to increasing torsional disorder in the polymer chain.

To conclude, we have measured the dynamics of the complex photoconductivity in MEH-PPV using the optical technique of THz-TDS. Monitoring of both the real and imaginary components of conductivity allows both free and bound excited charges to be probed simultaneously. We find a branching ratio of exciton vs charge generation of $\sim 10^{2}$. The small number of free charges relaxes on a subpicosecond time scale, while the primary exciton species persists over longer times. Our results are consistent with ultrafast charge generation through hot exciton dissociation.

The authors thank F. Wang, T. F. Heinz, A.W. Kleyn, and E. E. van Faassen for helpful discussions and R. C.V. van Schie and P. Schakel for excellent technical support. This work is financially supported by the Netherlands Organisation for Scientific Research (NWO) through the Foundation for Fundamental Research on Matter (FOM).

*Email address: e.hendry@chem.leidenuniv.nl

[1] M. Angelopoulos, IBM J. Res. Dev. 45, 57 (2001).

[2] R. H. Friend et al., Nature (London) 397, 121 (1999).

[3] N. Tessler et al., Nature (London) 382, 695 (1996).

[4] G. Hadziioannou and P. F.v. Hutten, Semiconducting Polymers (Wiley-VCH, Weinheim, 2000).

[5] A. Kohler et al., Nature (London) 392, 903 (1998).

[6] B. J. Schwartz, Annu. Rev. Phys. Chem. 54, 141 (2003).

[7] D. Moses et al., Phys. Rev. B 54, 4748 (1996).

[8] C. Silva et al., Phys. Rev. B 64, 125211 (2001).

[9] G. J. Denton et al., Synth. Met. 102, 1008 (1999).

[10] D. Moses et al., Phys. Rev. B 61, 9373 (2000).

[11] B. Kraabel et al., Phys. Rev. B 61, 8501 (2000).

[12] M. Yan et al., Phys. Rev. Lett. 72, 1104 (1994).

[13] S. Stagira et al., Phys. Rev. B 64, 205205 (2001).

[14] M. C. Beard et al., J. Phys. Chem. B 106, 7146 (2002).

[15] G. H. Gelinck and J. M. Warman, Chem. Phys. Lett. 277, 361 (1997).

[16] P. Jepsen et al., Appl. Phys. Lett. 79, 1291 (2001).

[17] E. Frankevich et al., Phys. Rev. B 62, 2505 (2000).

[18] H. D. Burrows et al., J. Mol. Struct. 563, 41 (2001).

[19] M. C. Beard et al., Phys. Rev. B 62, 15764 (2000).

[20] From the thermo-optical coefficient $\sim 10^{-4} \mathrm{~K}^{-1}$ [typical for polymers: E. Kang, J.Y. Bae, and B.S. Bae, J. Solgel Sc. Tech. 26, 981 (2003)] an estimated maximum pumpinduced temperature increase $\sim 100 \mathrm{~K}$ would cause a modulation $<0.001 \%$, much lower than the noise level of the experiment.

[21] F. C. Grozema et al., J. Phys. Chem. B 106, 7791 (2002).

[22] R. Hoofman et al., Nature (London) 392, 54 (1998).

[23] The GHz mobility was reported in J. M. Warman et al., J. Phys. C: Solid State Phys. 14, 9935 (2002). The carrier density in these experiments was calculated assuming that all the energy deposited in the sample was used to generate charges. As this efficiency is generally much smaller than $100 \%$, this sets a conservative lower limit for the $\mathrm{GHz}$ carrier mobility.

[24] N.W. Ashcroft and N. D. Mermin, Solid State Physics (Saunders College, Fort Worth, 1976), p. 542.

[25] G. H. Gelinck et al., Phys. Rev. B 62, 1489 (2000).

[26] J.W. v. d. Horst et al., Chem. Phys. Lett. 334, 303 (2001).

[27] M. Knupfer and J. Fink, Synth. Met. 141, 21 (2004).

[28] D. Moses et al., Synth. Met. 125, 93 (2001).

[29] J. A. E. Wasey et al., Phys. Rev. B 64, 205201 (2001).

[30] N. T. Harrison et al., Phys. Rev. Lett. 77, 1881 (1996).

[31] V. I. Arkhipov et al., Phys. Rev. Lett. 82, 1321 (1999).

[32] D. M. Basco and E. M. Conwell, Phys. Rev. B 66, 155210 (2002). 